\title{
Action de Hopf sur les opérateurs de Hecke modulaires tordus
}

\begin{abstract}
Abhishek Banerjee
Résumé. Soit $\Gamma \subseteq S L_{2}(\mathbb{Z})$ un sous-groupe principal de congruence. Pour chaque $\sigma \in S L_{2}(\mathbb{Z})$, nous introduisons l'ensemble $\mathcal{A}_{\sigma}(\Gamma)$ des opérateurs de Hecke modulaires tordus par $\sigma$. Alors, $\mathcal{A}_{\sigma}(\Gamma)$ est un $\mathcal{A}(\Gamma)$-module à droite, où $\mathcal{A}(\Gamma)$ est l'algèbre des opérateurs de Hecke modulaires introduite par Connes et Moscovici. En utilisant l'action d'une algèbre de Hopf ho sur $\mathcal{A}_{\sigma}(\Gamma)$, nous définissons les crochets de Rankin-Cohen réduits sur $\mathcal{A}_{\sigma}(\Gamma)$. De plus, $\mathcal{A}_{\sigma}(\Gamma)$ est muni d'une action de $\mathcal{H}_{1}$, où $\mathcal{H}_{1}$ est l'algèbre de Hopf des feuilletages de codimension 1 . Enfin, nous considérons les opérateurs entre les niveaux $\mathcal{A}_{\sigma}(\Gamma), \sigma \in S L_{2}(\mathbb{Z})$. Nous montrons que l'action de ces opérateurs peut être exprimée en termes d'une algèbre de Hopf $\mathfrak{h}_{\mathbb{Z}}$.
\end{abstract}

\begin{abstract}
Let $\Gamma \subseteq S L_{2}(\mathbb{Z})$ be a principal congruence subgroup. For each $\sigma \in S L_{2}(\mathbb{Z})$, we introduce the collection $\mathcal{A}_{\sigma}(\Gamma)$ of modular Hecke operators twisted by $\sigma$. Then, $\mathcal{A}_{\sigma}(\Gamma)$ is a right $\mathcal{A}(\Gamma)$-module, where $\mathcal{A}(\Gamma)$ is the modular Hecke algebra introduced by Connes and Moscovici. Using the action of a Hopf algebra $\mathfrak{h}_{0}$ on $\mathcal{A}_{\sigma}(\Gamma)$, we define reduced Rankin-Cohen brackets on $\mathcal{A}_{\sigma}(\Gamma)$. Moreover $\mathcal{A}_{\sigma}(\Gamma)$ carries an action of $\mathcal{H}_{1}$, where $\mathcal{H}_{1}$ is the Hopf algebra of foliations of codimension 1. Finally, we consider operators between the levels $\mathcal{A}_{\sigma}(\Gamma), \sigma \in S L_{2}(\mathbb{Z})$. We show that the action of these operators can be expressed in terms of a Hopf algebra $\mathfrak{h}_{\mathbb{Z}}$.
\end{abstract}

Mathematics Subject Classification (2010). 11F03, $16 \mathrm{~T} 05$.

Keywords. Modular Hecke algebras, Rankin-Cohen brackets, Hopf actions.

\section{Introduction}

Soit $\Gamma=\Gamma(N) \subseteq S L_{2}(\mathbb{Z})$ un sous-groupe principal de congruence. Alors, en combinant la structure multiplicative sur les formes modulaires avec l'action classique des opérateurs de Hecke, Connes et Moscovici [5] ont introduit l'algèbre $\mathcal{A}(\Gamma)$ des opérateurs de Hecke modulaires. De plus, on a une algèbre de Hopf $\mathcal{H}_{1}$ agissant sur $\mathcal{A}(\Gamma)$ telle que l'action de $\mathcal{H}_{1}$ est déterminée par certains opérateurs classiques sur les formes modulaires. L'algèbre de Hopf $\mathcal{H}_{1}$ fait partie d'une famille $\left\{\mathcal{H}_{n} \mid n \geq 1\right\}$ des algèbres de Hopf introduite dans [4] pour décrire certains opérateurs apparaissant dans la théorie des feuilletages. En particulier, $\mathcal{H}_{1}$ est appelée comme l'algèbre de Hopf des feuilletages de codimension 1.

Dans cet article, nous introduisons l'ensemble $\mathcal{A}_{\sigma}(\Gamma)$ des opérateurs de Hecke 
modulaires tordus par un élément $\sigma \in S L_{2}(\mathbb{Z})$. Comme dans le cas des opérateurs de Hecke modulaires, les opérateurs tordus dans $\mathcal{A}_{\sigma}(\Gamma)$ agissent sur les formes modulaires de niveau $\Gamma$. En particulier, quand $\sigma=1$, on a $\mathcal{A}_{\sigma}(\Gamma)=\mathcal{A}(\Gamma)$. En général, $\mathcal{A}_{\sigma}(\Gamma)$ est un $\mathcal{A}(\Gamma)$-module à droite. De plus, $\mathcal{A}_{\sigma}(\Gamma)$ est muni d'un appariement (voir (2.11))

$$
\left(\_, \_\right): \mathcal{A}_{\sigma}(\Gamma) \otimes \mathcal{A}_{\sigma}(\Gamma) \longrightarrow \mathcal{A}_{\sigma}(\Gamma)
$$

Soit $\mathfrak{l}_{0}$ l'algèbre de Lie engendrée par $\{X, Y\}$ modulo la relation $[Y, X]=X$ et soit $\mathfrak{h}_{0}$ l'algèbre enveloppante de $\mathfrak{l}_{0}$. Pour $h \in \mathfrak{h}_{0}$, posons $\Delta(h)=\sum h_{(1)} \otimes h_{(2)}$, où $\Delta: \mathfrak{h}_{0} \longrightarrow \mathfrak{h}_{0} \otimes \mathfrak{h}_{0}$ est le coproduit sur $\mathfrak{h}_{0}$. Alors, nous montrons que $\mathcal{A}_{\sigma}(\Gamma)$ est un module sur $\mathfrak{h}_{0}$ et on a (voir (2.25))

$$
h(F, G)=\sum\left(h_{(1)}(F), h_{(2)}(G)\right) \quad \forall h \in \mathfrak{h}_{0}, F, G \in \mathcal{A}_{\sigma}(\Gamma)
$$

De plus, nous montrons que $\mathcal{A}_{\sigma}(\Gamma)$ est muni d'une action de $\mathcal{H}_{1}$ et on a (voir (2.45))

$$
h(F * G)=\sum h_{(1)}(F) * h_{(2)}(G) \quad \forall h \in \mathcal{H}_{1}, F \in \mathcal{A}_{\sigma}(\Gamma), G \in \mathcal{A}(\Gamma)
$$

où le coproduit $\Delta: \mathcal{H}_{1} \longrightarrow \mathcal{H}_{1} \otimes \mathcal{H}_{1}$ sur $\mathcal{H}_{1}$ est donné par $\Delta(h)=\sum h_{(1)} \otimes h_{(2)}$ pour chaque $h \in \mathcal{H}_{1}$. Dans [10], l'action de $\mathfrak{h}_{0}$ sur l'algèbre $\mathcal{A}(\Gamma)$ est utilisée pour définir les crochets de Rankin-Cohen réduits sur $\mathcal{A}(\Gamma)$. Donc, en combinant l'appariement dans (1.1) avec l'action de $\mathfrak{h}_{0}$ sur $\mathcal{A}_{\sigma}(\Gamma)$, on définit les crochets de Rankin-Cohen réduits $\mathcal{R C}_{n}: \mathcal{A}_{\sigma}(\Gamma) \otimes \mathcal{A}_{\sigma}(\Gamma) \longrightarrow \mathcal{A}_{\sigma}(\Gamma), \forall n \geq 0$.

Puis, nous considérons les opérateurs entre les niveaux $\mathcal{A}_{\sigma}(\Gamma), \sigma \in S L_{2}(\mathbb{Z})$. Plus précisément, nous définissons un opérateur (voir (3.2))

$$
X_{\tau}: \mathcal{A}_{\sigma}(\Gamma) \longrightarrow \mathcal{A}_{\tau \sigma}(\Gamma)
$$

pour chaque $\tau, \sigma \in S L_{2}(\mathbb{Z})$. Soit $\rho_{m}:=\left(\begin{array}{cc}1 & m \\ 0 & 1\end{array}\right)$ pour chaque $m \in \mathbb{Z}$ et posons $\sigma(m)=\rho_{m} \cdot \sigma$. Alors, nous considérons le module gradué suivant :

$$
\mathbb{A}_{\sigma}(\Gamma):=\bigoplus_{n \in \mathbb{Z}} \mathcal{A}_{\sigma(n)}(\Gamma)
$$

et nous étudions les opérateurs $X_{m}: \mathcal{A}_{\sigma(n)}(\Gamma) \longrightarrow \mathcal{A}_{\sigma(m+n)}(\Gamma)$ entre les niveaux de la tour $\mathbb{A}_{\sigma}(\Gamma)$, où $X_{m}:=X_{\rho_{m}}, \forall m \in \mathbb{Z}$ dans le sens du (1.4). Donc, nous montrons que $\mathbb{A}_{\sigma}(\Gamma)$ est munie d'une action de l'algèbre de Lie $\mathfrak{l}_{\mathbb{Z}} \supseteq \mathfrak{l}_{0}$, engendrée $\operatorname{par}\left\{Z, X_{m} \mid m \in \mathbb{Z}\right\}$ modulo les relations suivantes (voir (3.7))

$$
\left[Z, X_{m}\right]=(m+1) X_{m} \quad\left[X_{m}, X_{m^{\prime}}\right]=0 \quad \forall m, m^{\prime} \in \mathbb{Z}
$$

De plus, nous généralisons l'appariement (1.1) sur $\mathcal{A}_{\sigma}(\Gamma)$ à un appariement (voir Proposition 3.3)

$$
(\ldots, \ldots): \mathcal{A}_{\tau_{1} \sigma}(\Gamma) \otimes \mathcal{A}_{\tau_{2} \sigma}(\Gamma) \longrightarrow \mathcal{A}_{\tau_{1} \tau_{2} \sigma}(\Gamma)
$$

où $\tau_{1}, \tau_{2} \in S L_{2}(\mathbb{Z})$ sont matrices telles que $\tau_{1} \tau_{2}=\tau_{2} \tau_{1}$. 
En particulier, on a un appariement $(\ldots, \ldots): \mathcal{A}_{\sigma(n)}(\Gamma) \otimes \mathcal{A}_{\sigma\left(n^{\prime}\right)}(\Gamma) \longrightarrow$ $\mathcal{A}_{\sigma\left(n+n^{\prime}\right)}(\Gamma), \forall n, n^{\prime} \in \mathbb{Z}$ et donc un appariement induit sur $\mathbb{A}_{\sigma}(\Gamma)$. Soit $\mathfrak{h}_{\mathbb{Z}}$ l'algèbre enveloppante de $\mathfrak{l}_{\mathbb{Z}}$. Enfin, nous montrons que l'appariement sur $\mathbb{A}_{\sigma}(\Gamma)$ induit par (1.7) se comporte bien vis-à-vis de l'action de $\mathfrak{h}_{\mathbb{Z}}$. Autrement dit, on a (voir (3.17))

$$
h(F, G)=\sum\left(h_{(1)}(F), h_{(2)}(G)\right) \quad \forall h \in \mathfrak{h}_{\mathbb{Z}}, F, G \in \mathbb{A}_{\sigma}(\Gamma)
$$

où le coproduit $\Delta: \mathfrak{h}_{\mathbb{Z}} \longrightarrow \mathfrak{h}_{\mathbb{Z}} \otimes \mathfrak{h}_{\mathbb{Z}}$ sur $\mathfrak{h}_{\mathbb{Z}}$ est donné par $\Delta(h):=\sum h_{(1)} \otimes h_{(2)}$ pour chaque $h \in \mathfrak{h}_{\mathbb{Z}}$.

Remerciements. Je tiens à remercier sincèrement Prof. Alain Connes pour ses conseils. De plus, une grande partie de cet article a été écrit lors d'un séjour à l'Isaac Newton Institute for Mathematical Sciences, Cambridge, Royaume-Uni. Je souhaite remercier l'Institut pour son hospitalité.

\section{Les opérateurs de Hecke modulaires tordus}

Pour chaque entier $M \geq 1$, nous notons par $\Gamma(M)$ le sous-groupe principal de congruence de niveau $M$. Si $\gamma=\left(\begin{array}{ll}a & b \\ c & d\end{array}\right) \in G L_{2}^{+}(\mathbb{Q})$, on définit une fonction :

$$
j_{\gamma}: \mathbb{H} \longrightarrow \mathbb{C} \quad j_{\gamma}(z):=(c z+d)^{-1} .
$$

où $\mathbb{H}$ est le demi-plan de Poincaré. On dispose d'une action de $G L_{2}^{+}(\mathbb{Q})$ sur $\mathbb{H}$ comme suit :

$$
\left(\begin{array}{ll}
a & b \\
c & d
\end{array}\right) \cdot z:=\frac{a z+b}{c z+d} \quad \forall\left(\begin{array}{ll}
a & b \\
c & d
\end{array}\right) \in G L_{2}^{+}(\mathbb{Q}), z \in \mathbb{H}
$$

Une fonction holomorphe $f: \mathbb{H} \longrightarrow \mathbb{C}$ est dite une forme modulaire de niveau $M$ et poids $k \in \mathbb{Z}$ si elle satisfait

$$
f(z)=\left(\left.f\right|_{k} \gamma\right)(z):=f(\gamma z) j_{\gamma}(z)^{k} \quad \forall \gamma \in \Gamma(M), z \in \mathbb{H}
$$

et $f$ est «holomorphe aux pointes» (voir, par exemple, [9]). Nous notons par $\mathcal{M}_{k}(\Gamma(M))$ l'ensemble des formes modulaires de niveau $M$ et poids $k$. Nous posons

$$
\mathcal{M}(\Gamma(M)):=\bigoplus_{k \geq 0} \mathcal{M}_{k}(\Gamma(M)) \quad \mathcal{M}:=\lim _{M \geq 1} \mathcal{M}(\Gamma(M))
$$

De plus, une forme modulaire $f$ est dite cuspidale si $f$ est nulle aux pointes (voir [9] pour les définitions). Notons par $\mathcal{M}_{k}^{0}(\Gamma(M))$ l'ensemble des formes modulaires cuspidales de niveau $M$ et poids $k$ et posons

$$
\mathcal{M}^{0}(\Gamma(M)):=\bigoplus_{k \geq 0} \mathcal{M}_{k}^{0}(\Gamma(M)) \quad \mathcal{M}^{0}:=\underset{\overrightarrow{M \geq 1}}{\lim _{\longrightarrow}} \mathcal{M}^{0}(\Gamma(M)) .
$$


Alors, $\mathcal{M}^{0}$ est un idéal de l'anneau $\mathcal{M}$. Nous rappelons ici la notion d'un opérateur de Hecke modulaire introduit par Connes et Moscovici [5, § 1].

Définition 2.1. Soit $\Gamma$ un sous-groupe principal de congruence de $S L_{2}(\mathbb{Z})$. Un opérateur de Hecke modulaire de niveau $\Gamma$ est une fonction à support fini

$$
F: \Gamma \backslash G L_{2}^{+}(\mathbb{Q}) \longrightarrow \mathcal{M} \quad \Gamma \alpha \mapsto F_{\alpha} \in \mathcal{M}
$$

telle qu'elle satisfait la condition suivante :

$$
F_{\alpha \gamma}=F_{\alpha} \mid \gamma \quad \forall \gamma \in \Gamma
$$

Notons par $\mathcal{A}(\Gamma)$ l'ensemble des opérateurs de Hecke modulaires de niveau $\Gamma$.

Soit $\Gamma$ un sous-groupe principal de congruence de $S L_{2}(\mathbb{Z})$. Pour chaque $\sigma \in S L_{2}(\mathbb{Z})$, nous sommes prêts à introduire les opérateurs de Hecke modulaires (de niveau $\Gamma$ ) tordus par $\sigma$.

Définition 2.2. Fixons $\sigma \in S L_{2}(\mathbb{Z})$. Soit $\Gamma$ un sous-groupe principal de congruence de $S L_{2}(\mathbb{Z})$. Un opérateur de Hecke modulaire (de niveau $\Gamma$ ) tordu par $\sigma$ est une fonction à support fini

$$
F: \Gamma \backslash G L_{2}^{+}(\mathbb{Q}) \longrightarrow \mathcal{M} \quad \Gamma \alpha \mapsto F_{\alpha} \in \mathcal{M}
$$

telle qu'elle satisfait la condition suivante:

$$
F_{\alpha \gamma}=F_{\alpha} \mid \sigma \gamma \sigma^{-1} \quad \forall \gamma \in \Gamma
$$

Notons par $\mathcal{A}_{\sigma}(\Gamma)$ l'ensemble des opérateurs de Hecke modulaires tordus par $\sigma . \mathrm{Si}$ $F \in \mathcal{A}_{\sigma}(\Gamma)$ est un opérateur tel que $F_{\alpha} \in \mathcal{M}^{0}, \forall \alpha \in G_{2}^{+}(\mathbb{Q})$, on dit que $F$ est cuspidal. Notons par $\mathcal{A}_{\sigma}^{0}(\Gamma)$ le sous-ensemble des opérateurs cuspidals.

Proposition 2.3. Soit $\Gamma$ un sous-groupe principal de congruence de $S L_{2}(\mathbb{Z})$. Fixons $\sigma \in S L_{2}(\mathbb{Z})$. Alors, on a un appariement

$$
\left(\_, \_\right): \mathcal{A}_{\sigma}(\Gamma) \otimes \mathcal{A}_{\sigma}(\Gamma) \longrightarrow \mathcal{A}_{\sigma}(\Gamma)
$$

défini comme suit :

$$
(F, G)_{\alpha}:=\sum_{\beta \in \Gamma \backslash S L_{2}(\mathbb{Z})} F_{\beta \sigma} \cdot G_{\alpha \sigma^{-1} \beta^{-1}} \mid \sigma \beta \quad \forall F, G \in \mathcal{A}_{\sigma}(\Gamma), \alpha \in G L_{2}^{+}(\mathbb{Q}) .
$$


Démonstration. Prenons $\gamma \in \Gamma$. Alors, pour chaque $\beta \in S L_{2}(\mathbb{Z})$, on a

$$
\begin{aligned}
F_{\gamma \beta \sigma} & =F_{\beta \sigma} \\
G_{\alpha \sigma^{-1} \beta^{-1} \gamma^{-1}} \mid \sigma \gamma \beta & =G_{\alpha \sigma^{-1} \beta^{-1}}\left|\sigma \gamma^{-1} \sigma^{-1} \sigma \gamma \beta=G_{\alpha \sigma^{-1} \beta^{-1}}\right| \sigma \beta
\end{aligned}
$$

et donc la somme dans (2.11) est bien définie. De plus, puisque $G \in \mathcal{A}_{\sigma}(\Gamma)$ est une fonction de $\Gamma \backslash G_{2}^{+}(\mathbb{Q})$ vers $\mathcal{M}$, on a $G_{\alpha \sigma^{-1} \beta^{-1}}=G_{\gamma \alpha \sigma^{-1} \beta^{-1}}$. Utilisant la définition de $(F, G)_{\alpha}$ dans (2.11), on voit que $(F, G)_{\gamma \alpha}=(F, G)_{\alpha}$. D'autre part, on a

$$
(F, G)_{\alpha \gamma}=\sum_{\beta \in \Gamma \backslash S L_{2}(\mathbb{Z})} F_{\beta \sigma} \cdot G_{\alpha \gamma \sigma^{-1} \beta^{-1}} \mid \sigma \beta
$$

Dans (2.13), nous posons $\delta=\beta \sigma \gamma^{-1} \sigma^{-1}$. Puisque $F \in \mathcal{A}_{\sigma}(\Gamma)$, on a $F_{\delta \sigma \gamma}=$ $F_{\delta \sigma} \mid \sigma \gamma \sigma^{-1}$. Alors, on peut écrire (2.13) comme

$$
\begin{aligned}
(F, G)_{\alpha \gamma} & =\sum_{\delta \in \Gamma \backslash S L_{2}(\mathbb{Z})} F_{\delta \sigma \gamma} \cdot G_{\alpha \sigma^{-1} \delta^{-1}} \mid \sigma \delta \sigma \gamma \sigma^{-1} \\
& =\sum_{\delta \in \Gamma \backslash S L_{2}(\mathbb{Z})}\left(F_{\delta \sigma} \mid \sigma \gamma \sigma^{-1}\right) \cdot\left(G_{\alpha \sigma^{-1} \delta^{-1}} \mid \sigma \delta\right) \mid \sigma \gamma \sigma^{-1} \\
& =\left(\sum_{\delta \in \Gamma \backslash S L_{2}(\mathbb{Z})} F_{\delta \sigma} \cdot G_{\alpha \sigma^{-1} \delta^{-1}} \mid \sigma \delta\right) \mid \sigma \gamma \sigma^{-1} \\
& =(F, G)_{\alpha} \mid \sigma \gamma \sigma^{-1}
\end{aligned}
$$

Donc, $(F, G) \in \mathcal{A}_{\sigma}(\Gamma)$.

Ici, nous remarquons que la formule (2.11) ne définit pas la structure d'une algèbre $\operatorname{sur} \mathcal{A}_{\sigma}(\Gamma)$. En fait, pour $F, G, H \in \mathcal{A}_{\sigma}(\Gamma)$, l'opérateur $((F, G), H) \in \mathcal{A}_{\sigma}(\Gamma)$ n' est pas nécessairement égal à $(F,(G, H))$.

Quand $\sigma=1$, il est clair que $\mathcal{A}_{\sigma}(\Gamma)=\mathcal{A}(\Gamma)$, où $\mathcal{A}(\Gamma)$ est l'ensemble des opérateurs de Hecke modulaires introduit par Connes et Moscovici $[5, \S 1]$. Nous montrons que les opérateurs de Hecke modulaires tordus agissent sur $\mathcal{M}(\Gamma)$. Quand $\sigma=1$, cette action est la même que l'action de $\mathcal{A}(\Gamma)$ sur $\mathcal{M}(\Gamma)$ (voir [5]).

Proposition 2.4. Soit $\Gamma$ un sous-groupe principal de congruence de $S L_{2}(\mathbb{Z})$. Fixons $\sigma \in S L_{2}(\mathbb{Z})$. Alors, les opérateurs de Hecke modulaires tordus par $\sigma$ agissent sur $\mathcal{M}(\Gamma)$ comme suit :

$$
F * f:=\sum_{\alpha \in \Gamma \backslash G L_{2}^{+}(\mathbb{Q})} F_{\alpha \sigma} \cdot f \mid \alpha \quad \forall F \in \mathcal{A}_{\sigma}(\Gamma), f \in \mathcal{M}(\Gamma)
$$

En particulier, si $f \in \mathcal{M}^{0}(\Gamma) \subseteq \mathcal{M}(\Gamma), F * f \in \mathcal{M}^{0}(\Gamma)$ pour chaque $F \in \mathcal{A}_{\sigma}(\Gamma)$. 
Démonstration. Prenons $\gamma \in \Gamma$. Alors, on a :

$$
\begin{aligned}
(F * f) \mid \gamma & =\sum_{\alpha \in \Gamma \backslash G L_{2}^{+}(\mathbb{Q})}\left(F_{\alpha \sigma} \cdot f \mid \alpha\right) \mid \gamma \\
& =\sum_{\alpha \in \Gamma \backslash G L_{2}^{+}(\mathbb{Q})}\left(F_{\alpha \sigma} \mid \gamma\right) \cdot(f \mid \alpha \gamma) \\
& =\sum_{\alpha \in \sigma^{-1} \gamma \sigma} \cdot f \mid \alpha \gamma \\
\alpha & F_{\alpha \backslash G L_{2}^{+}(\mathbb{Q})} \\
& =\sum_{\alpha \in \Gamma \backslash G L_{2}^{+}(\mathbb{Q})} F_{\alpha \gamma \sigma} \cdot f \mid \alpha \gamma=F * f
\end{aligned}
$$

Donc, $F * f \in \mathcal{M}(\Gamma)$. Enfin, supposons que $f \in \mathcal{M}^{0}(\Gamma)$. Alors, $\mathcal{M}^{0}(\Gamma)$ étant un idéal de l'anneau $\mathcal{M}(\Gamma)$, il s'ensuit que

$$
(F * f)=\sum_{\alpha \in \Gamma \backslash G L_{2}^{+}(\mathbb{Q})} F_{\alpha \sigma} \cdot f \mid \alpha \in \mathcal{M}^{0}(\Gamma) .
$$

Soit $k \in \mathbb{Z}$. Alors, on a un opérateur différentiel (voir [5, § 3]) :

$$
X: \mathcal{M}_{k} \longrightarrow \mathcal{M}_{k+2}, \quad X:=\frac{1}{2 \pi i} \frac{d}{d z}-\frac{1}{12 \pi i} \frac{d}{d z}(\log \Delta(z)) \cdot Y
$$

où $Y: \mathcal{M}_{k} \longrightarrow \mathcal{M}_{k}$ est définie comme $Y(f):=(k / 2) \cdot f, \forall f \in \mathcal{M}_{k}$ et $\Delta(z)$ est la forme modulaire (de poids 12) suivante :

$$
\Delta(z):=(2 \pi)^{12} q \prod_{n=1}^{\infty}\left(1-q^{n}\right)^{24}, \quad q=e^{2 \pi i z}, z \in \mathbb{H}
$$

Si $\alpha \in S L_{2}(\mathbb{Z})$, on peut vérifier que $X\left(\left.f\right|_{k} \alpha\right)=\left.X(f)\right|_{k+2} \alpha$. Plus généralement, pour $\alpha \in G L_{2}^{+}(\mathbb{Q})$, on a (voir [5, Lemma 5])

$$
X\left(\left.f\right|_{k} \alpha\right)=X(f)_{k+2}\left|\alpha-\left(\mu_{\alpha^{-1}} \cdot Y(f)\right)\right|_{k+2} \alpha
$$

où

$$
\mu_{\delta}:=\frac{1}{12 \pi i} \frac{d}{d z} \log \frac{\Delta \mid \delta}{\Delta} \quad \forall \delta \in G L_{2}^{+}(\mathbb{Q})
$$

En particulier, $\mu_{\alpha}=0, \forall \alpha \in S L_{2}(\mathbb{Z})$. De plus, on peut vérifier que (voir [5, (3.12)])

$$
\mu_{\alpha_{1} \alpha_{2}}=\mu_{\alpha_{1}} \mid \alpha_{2}+\mu_{\alpha_{2}} \quad \forall \alpha_{1}, \alpha_{2} \in G L_{2}^{+}(\mathbb{Q})
$$

Puisque $0=\mu_{1}=\mu_{\alpha^{-1} \alpha}$, il résulte de (2.21) qu'on peut écrire (2.19) comme

$$
X\left(\left.f\right|_{k} \alpha\right)=X(f)_{k+2} \mid \alpha+\mu_{\alpha} \cdot\left(\left.Y(f)\right|_{k} \alpha\right) .
$$


Enfin, notons que $X, Y$ sont des dérivations sur $\mathcal{M}$ et $[Y, X]=X$ (voir $[5, \S 3])$. Soit $\mathfrak{h}_{0}$ l'algèbre enveloppante de l'algèbre de Lie $\mathfrak{l}_{0}$ engendrée par $\{X, Y\}$ modulo la relation $[Y, X]=X$. Alors, le coproduit $\Delta: \mathfrak{h}_{0} \longrightarrow \mathfrak{h}_{0} \otimes \mathfrak{h}_{0}$ sur l'algèbre de Hopf $\mathfrak{h}_{0}$ est défini par

$$
\Delta(X)=X \otimes 1+1 \otimes X \quad \Delta(Y)=Y \otimes 1+1 \otimes Y .
$$

Proposition 2.5. Soit $\Gamma$ un sous-groupe principal de congruence de $S L_{2}(\mathbb{Z})$. Fixons $\sigma \in S L_{2}(\mathbb{Z})$. Pour chaque $h \in \mathfrak{h}_{0}$, posons $\Delta(h):=\sum h_{(1)} \otimes h_{(2)}$.

(a) Alors, $\mathcal{A}_{\sigma}(\Gamma)$ est un module sur $\mathfrak{h}_{0}:$ pour $F \in \mathcal{A}_{\sigma}(\Gamma), \alpha \in G L_{2}^{+}(\mathbb{Q})$, on a

$$
X(F)_{\alpha}=X\left(F_{\alpha}\right) \quad Y(F)_{\alpha}=Y\left(F_{\alpha}\right) \quad \forall \alpha \in G L_{2}^{+}(\mathbb{Q})
$$

(b) L'algèbre $\mathfrak{h}_{0} a$ «une action de Hopf » sur l'appariement $(\ldots, \ldots): \mathcal{A}_{\sigma}(\Gamma) \otimes$ $\mathcal{A}_{\sigma}(\Gamma) \longrightarrow \mathcal{A}_{\sigma}(\Gamma)$. Autrement dit, pour $F, G \in \mathcal{A}_{\sigma}(\Gamma)$, on $a:$

$$
\sum\left(h_{(1)}(F), h_{(2)}(G)\right)=h(F, G)
$$

Démonstration. (a) Pour montrer qu'on a une action de $\mathfrak{h}_{0}$ sur $\mathcal{A}_{\sigma}(\Gamma)$, il suffit de vérifier la relation $[Y, X]=X$ entre les opérateurs dans (2.24). Soit $F \in \mathcal{A}_{\sigma}(\Gamma)$. Alors, pour $\alpha \in G L_{2}^{+}(\mathbb{Q})$, on a :

$$
[Y, X](F)_{\alpha}=[Y, X]\left(F_{\alpha}\right)=X\left(F_{\alpha}\right)=X(F)_{\alpha}
$$

(b) Il suffit de vérifier (2.25) pour les générateurs $X, Y$. Pour $F, G \in \mathcal{A}_{\sigma}(\Gamma)$ et $\alpha \in G L_{2}^{+}(\mathbb{Q})$, on a

$$
\begin{aligned}
(X(F, G))_{\alpha} & =X\left((F, G)_{\alpha}\right) \\
& =\sum_{\beta \in \Gamma \backslash S L_{2}(\mathbb{Z})} X\left(F_{\beta \sigma} \cdot\left(G_{\alpha \sigma^{-1} \beta^{-1}} \mid \sigma \beta\right)\right) \\
& =\sum_{\beta \in \Gamma \backslash S L_{2}(\mathbb{Z})} X\left(F_{\beta \sigma}\right) \cdot\left(G_{\alpha \sigma^{-1} \beta^{-1}} \mid \sigma \beta\right)+\sum_{\beta \in \Gamma \backslash S L_{2}(\mathbb{Z})} F_{\beta \sigma} \cdot X\left(G_{\alpha \sigma^{-1} \beta^{-1}} \mid \sigma \beta\right) \\
& =(X(F), G)_{\alpha}+\sum_{\beta \in \Gamma \backslash S L_{2}(\mathbb{Z})} F_{\beta \sigma} \cdot X\left(G_{\alpha \sigma^{-1} \beta^{-1}}\right) \mid \sigma \beta \\
& =(X(F), G)_{\alpha}+(F, X(G))_{\alpha}
\end{aligned}
$$

De même, on peut vérifier que $(Y(F, G))_{\alpha}=(Y(F), G)_{\alpha}+(F, Y(G))_{\alpha}$.

Soient $G, H \in \mathcal{A}(\Gamma)$. Rappelons que le produit sur l'algèbre $\mathcal{A}(\Gamma)$ est défini comme (voir [5, Proposition 2]) :

$$
(G * H)_{\alpha}:=\sum_{\beta \in \Gamma \backslash G L_{2}^{+}(\mathbb{Q})} G_{\beta} \cdot H_{\alpha \beta^{-1}} \mid \beta \quad \forall \alpha \in G L_{2}^{+}(\mathbb{Q}) .
$$


Pour chaque $\alpha \in G L_{2}^{+}(\mathbb{Q})$, notons qu'on peut exprimer $(G * H)_{\alpha}$ comme suit :

$$
(G * H)_{\alpha}:=\sum_{\alpha_{2} \alpha_{1}=\alpha} G_{\alpha_{1}} \cdot H_{\alpha_{2}} \mid \alpha_{1}
$$

où la somme dans (2.28) est prise sur tous les couples $\left(\alpha_{1}, \alpha_{2}\right)$ tels que $\alpha_{2} \alpha_{1}=\alpha$ modulo la relation d'équivalence :

$$
\left(\alpha_{1}, \alpha_{2}\right) \sim\left(\gamma \alpha_{1}, \alpha_{2} \gamma^{-1}\right) \quad \forall \gamma \in \Gamma
$$

Pour chaque $\sigma \in S L_{2}(\mathbb{Z})$, nous montrons que $\mathcal{A}_{\sigma}(\Gamma)$ est un $\mathcal{A}(\Gamma)$-module à droite.

Proposition 2.6. Soit $\Gamma$ un sous-groupe principal de congruence de $S L_{2}(\mathbb{Z})$. Fixons $\sigma \in S L_{2}(\mathbb{Z})$. Alors, $\mathcal{A}_{\sigma}(\Gamma)$ est muni de la structure d'un $\mathcal{A}(\Gamma)$-module à droite :

$$
(F * G)_{\alpha}:=\sum_{\beta \in \Gamma \backslash G L_{2}^{+}(\mathbb{Q})} F_{\beta \sigma} \cdot G_{\alpha \sigma^{-1} \beta^{-1}} \mid \beta \quad \forall F \in \mathcal{A}_{\sigma}(\Gamma), G \in \mathcal{A}(\Gamma), \alpha \in G L_{2}^{+}(\mathbb{Q})
$$

De plus, $\mathcal{A}_{\sigma}^{0}(\Gamma)$ est un $\mathcal{A}(\Gamma)$ sous-module de $\mathcal{A}_{\sigma}(\Gamma)$.

Démonstration. Prenons $\gamma \in \Gamma$. Alors, on a

$$
\begin{aligned}
F_{\gamma \beta \sigma} & =F_{\beta \sigma} \\
G_{\alpha \sigma^{-1} \beta^{-1} \gamma^{-1}} \mid \gamma \beta & =G_{\alpha \sigma^{-1} \beta^{-1}}\left|\gamma^{-1} \gamma \beta=G_{\alpha \sigma^{-1} \beta^{-1}}\right| \beta
\end{aligned}
$$

et donc la somme dans (2.30) est bien définie. De plus, comme dans la démonstration de Proposition 2.3, on peut vérifier que $(F * G)_{\gamma \alpha}=(F * G)_{\alpha}$. Par définition, on a

$$
(F * G)_{\alpha \gamma}=\sum_{\beta \in \Gamma \backslash G_{2}^{+}(\mathbb{Q})} F_{\beta \sigma} \cdot G_{\alpha \gamma \sigma^{-1} \beta^{-1}} \mid \beta
$$

En posant $\delta=\beta \sigma \gamma^{-1} \sigma^{-1}$ et utilisant le fait que $F_{\delta \sigma \gamma}=F_{\delta \sigma} \mid \sigma \gamma \sigma^{-1}$, on a

$$
\begin{aligned}
(F * G)_{\alpha \gamma} & =\sum_{\delta \in \Gamma \backslash G_{2}^{+}(\mathbb{Q})} F_{\delta \sigma \gamma} \cdot G_{\alpha \sigma^{-1}} \delta^{-1} \mid \delta \sigma \gamma \sigma^{-1} \\
& =\sum_{\delta \in \Gamma \backslash G_{2}^{+}(\mathbb{Q})}\left(F_{\delta \sigma} \mid \sigma \gamma \sigma^{-1}\right) \cdot\left(G_{\alpha \sigma^{-1} \delta^{-1}} \mid \delta\right) \mid \sigma \gamma \sigma^{-1} \\
& =\left(\sum_{\delta \in \Gamma \backslash G_{2}^{+}(\mathbb{Q})} F_{\delta \sigma} \cdot G_{\alpha \sigma^{-1} \delta^{-1}} \mid \delta\right) \mid \sigma \gamma \sigma^{-1} \\
& =(F * G)_{\alpha} \mid \sigma \gamma \sigma^{-1}
\end{aligned}
$$

Il reste à montrer que $(F * G) * H=F *(G * H), \forall F \in \mathcal{A}_{\sigma}(\Gamma), G, H \in \mathcal{A}(\Gamma)$. 
Notons que

$$
(F * G)_{\alpha}=\sum_{\alpha_{2} \alpha_{1}=\alpha} F_{\alpha_{1}} \cdot G_{\alpha_{2}} \mid \alpha_{1} \sigma^{-1}
$$

où la somme dans (2.34) est prise sur tous les couples $\left(\alpha_{1}, \alpha_{2}\right)$ tels que $\alpha_{2} \alpha_{1}=\alpha$ modulo la relation d'équivalence :

$$
\left(\alpha_{1}, \alpha_{2}\right) \sim\left(\gamma \alpha_{1}, \alpha_{2} \gamma^{-1}\right) \quad \forall \gamma \in \Gamma
$$

Donc, on peut écrire :

$$
((F * G) * H)_{\alpha}=\sum_{\alpha_{3} \alpha_{2} \alpha_{1}=\alpha} F_{\alpha_{1}} \cdot\left(G_{\alpha_{2}} \mid \alpha_{1} \sigma^{-1}\right) \cdot\left(H_{\alpha_{3}} \mid \alpha_{2} \alpha_{1} \sigma^{-1}\right)
$$

où la somme dans (2.36) est prise sur tous les triplets $\left(\alpha_{1}, \alpha_{2}, \alpha_{3}\right)$ tels que $\alpha_{3} \alpha_{2} \alpha_{1}=\alpha$ modulo la relation d'équivalence :

$$
\left(\alpha_{1}, \alpha_{2}, \alpha_{3}\right) \sim\left(\gamma \alpha_{1}, \gamma^{\prime} \alpha_{2} \gamma^{-1}, \alpha_{3} \gamma^{\prime-1}\right) \quad \forall \gamma, \gamma^{\prime} \in \Gamma
$$

D'autre part, on a

$$
\begin{aligned}
(F *(G * H))_{\alpha} & =\sum_{\alpha_{2}^{\prime} \alpha_{1}=\alpha} F_{\alpha_{1}} \cdot(G * H)_{\alpha_{2}^{\prime}} \mid \alpha_{1} \sigma^{-1} \\
& =\sum_{\alpha_{3} \alpha_{2} \alpha_{1}=\alpha} F_{\alpha_{1}} \cdot\left(G_{\alpha_{2}} \cdot\left(H_{\alpha_{3}} \mid \alpha_{2}\right)\right) \mid \alpha_{1} \sigma^{-1} \\
& =\sum_{\alpha_{3} \alpha_{2} \alpha_{1}=\alpha} F_{\alpha_{1}} \cdot\left(G_{\alpha_{2}} \mid \alpha_{1} \sigma^{-1}\right) \cdot\left(H_{\alpha_{3}} \mid \alpha_{2} \alpha_{1} \sigma^{-1}\right)
\end{aligned}
$$

où la somme dans (2.38) est prise sur tous les triplets $\left(\alpha_{1}, \alpha_{2}, \alpha_{3}\right)$ tels que $\alpha_{3} \alpha_{2} \alpha_{1}=\alpha$, modulo la relation d'équivalence dans (2.37). Combinant (2.36) et (2.38), on voit que $((F * G) * H)_{\alpha}=(F *(G * H))_{\alpha}$.

Enfin, supposons que $F \in \mathcal{A}_{\sigma}^{0}(\Gamma)$. Donc, $F_{\beta \sigma} \in \mathcal{M}^{0}$ pour chaque $\beta \in G L_{2}^{+}(\mathbb{Q})$. Alors, il s'ensuit de (2.30) que $(F * G) \in \mathcal{A}_{\sigma}^{0}(\Gamma)$ pour chaque $G \in \mathcal{A}(\Gamma)$.

Soit $\mathcal{H}$ une algèbre de Hopf agissant sur une algèbre $\mathcal{A}$. On dit que l'action de $\mathcal{H}$ est plate si elle satisfait (voir, par exemple, $[7, \S 2.1]$ ) :

$$
h(a b)=\sum h_{(1)}(a) h_{(2)}(b) \quad \forall h \in \mathcal{H}, a, b \in \mathcal{A}
$$

où $\Delta(h)=\sum h_{(1)} \otimes h_{(2)}$ est le coproduit sur $\mathcal{H}$. Plus généralement, supposons que $\mathcal{H}$ agit sur $\mathcal{P}$ et $\mathcal{A}$, où $\mathcal{A}$ est une algèbre et $\mathcal{P}$ est un $\mathcal{A}$-module à droite. Alors, on dit que l'action de $\mathcal{H}$ sur $(\mathcal{P}, \mathcal{A})$ est plate si elle satisfait (voir [1, Definition 3.11])

$$
h(p a)=\sum h_{(1)}(p) h_{(2)}(a) \quad \forall h \in \mathcal{H}, p \in \mathcal{P}, a \in \mathcal{A} .
$$


Dans [5], Connes et Moscovici ont introduit une action de l'algèbre de Hopf $\mathcal{H}_{1}$ sur $\mathcal{A}(\Gamma)$. Alors, $\mathcal{H}_{1}$ fait partie d'une famille $\left\{\mathcal{H}_{n} \mid n \geq 1\right\}$ des algèbres de Hopf définie dans [4] et $\mathcal{H}_{1}$ est appelée comme l'algèbre de Hopf des feuilletages de codimension 1. Nous rappelons ici la définition de l'algèbre de Hopf $\mathcal{H}_{1}$. Soit $\mathcal{L}_{1}$ l'algèbre de Lie engendrée par les opérateurs $\left\{X, Y, \delta_{n} \mid n \geq 1\right\}$ modulo les relations suivantes :

$$
\begin{aligned}
& {[Y, X]=X, \quad\left[X, \delta_{n}\right]=\delta_{n+1}, \quad\left[Y, \delta_{n}\right]=n \delta_{n}, \quad\left[\delta_{k}, \delta_{l}\right]=0,} \\
& \forall n, k, l \geq 1
\end{aligned}
$$

Alors, $\mathcal{H}_{1}$ est définie comme l'algèbre enveloppante de $\mathcal{L}_{1}$. De plus, le coproduit $\Delta: \mathcal{H}_{1} \longrightarrow \mathcal{H}_{1} \otimes \mathcal{H}_{1}$ sur $\mathcal{H}_{1}$ est défini comme :

$$
\begin{gathered}
\Delta(X)=X \otimes 1+1 \otimes X+\delta_{1} \otimes Y \\
\Delta(Y)=Y \otimes 1+1 \otimes Y \quad \Delta\left(\delta_{1}\right)=\delta_{1} \otimes 1+1 \otimes \delta_{1} .
\end{gathered}
$$

L'antipode $S: \mathcal{H}_{1} \longrightarrow \mathcal{H}_{1}$ est donné par :

$$
S(X)=-X+\delta_{1} Y \quad S(Y)=-Y \quad S\left(\delta_{1}\right)=-\delta_{1} .
$$

Alors, $\mathcal{H}_{1}$ est une algèbre de Hopf (voir $[5, \S 2]$ ). Pour savoir plus sur les algèbres de Hopf $\mathcal{H}_{n}, n \geq 1$, voir [4]. Nous allons montrer que $\mathcal{H}_{1}$ agit sur $\mathcal{A}_{\sigma}(\Gamma)$. De plus, l'action de $\mathcal{H}_{1}$ sur $\left(\mathcal{A}_{\sigma}(\Gamma), \mathcal{A}(\Gamma)\right)$ est plate dans le sens du (2.40).

Proposition 2.7. Soit $\Gamma$ un sous-groupe principal de congruence de $S L_{2}(\mathbb{Z})$. Fixons $\sigma \in S L_{2}(\mathbb{Z})$.

(a) Alors, $\mathcal{A}_{\sigma}(\Gamma)$ est un module sur $\mathcal{H}_{1}$; pour $F \in \mathcal{A}_{\sigma}(\Gamma), \alpha \in G L_{2}^{+}(\mathbb{Q})$, on a

$$
\begin{array}{r}
X(F)_{\alpha}=X\left(F_{\alpha}\right) \quad Y(F)_{\alpha}=Y\left(F_{\alpha}\right) \quad \delta_{n}(F)_{\alpha}=X^{n-1}\left(\mu_{\alpha \sigma^{-1}}\right) \cdot F_{\alpha} \\
\forall n \geq 1 .
\end{array}
$$

En particulier, quand $\sigma=1$, l'action de $\mathcal{H}_{1}$ sur $\mathcal{A}_{\sigma}(\Gamma)=\mathcal{A}(\Gamma)$ est la même que l'action de $\mathcal{H}_{1}$ sur $\mathcal{A}(\Gamma)$ définie par Connes et Moscovici.

(b) Pour chaque $h \in \mathcal{H}_{1}$, posons $\Delta(h):=\sum h_{(1)} \otimes h_{(2)}$. Alors, l'action de $\mathcal{H}_{1}$ $\operatorname{sur}\left(\mathcal{A}_{\sigma}(\Gamma), \mathcal{A}(\Gamma)\right)$ est plate dans le sens du (2.40). Autrement dit, pour $F \in \mathcal{A}_{\sigma}(\Gamma), G \in \mathcal{A}(\Gamma)$, on $a$ :

$$
\sum h_{(1)}(F) * h_{(2)}(G)=h(F * G) .
$$

Démonstration. (a) Soit $\gamma \in \Gamma$. Utilisant (2.21), on a

$$
\mu_{\alpha \gamma \sigma^{-1}}=\mu_{\alpha \sigma^{-1}}\left|\sigma \gamma \sigma^{-1}+\mu_{\sigma \gamma \sigma^{-1}}=\mu_{\alpha \sigma^{-1}}\right| \sigma \gamma \sigma^{-1} .
$$


Alors, pour $F \in \mathcal{A}_{\sigma}(\Gamma)$, il résulte que

$$
\begin{aligned}
\delta_{n}(F)_{\alpha \gamma}=X^{n-1}\left(\mu_{\alpha \gamma \sigma^{-1}}\right) F_{\alpha \gamma} & =X^{n-1}\left(\mu_{\alpha \sigma^{-1}} \mid \sigma \gamma \sigma^{-1}\right)\left(F_{\alpha} \mid \sigma \gamma \sigma^{-1}\right) \\
& =\delta_{n}(F)_{\alpha} \mid \sigma \gamma \sigma^{-1}
\end{aligned}
$$

et donc $\delta_{n}(F) \in \mathcal{A}_{\sigma}(\Gamma)$ pour chaque $n \geq 1$. De plus, il est clair que $X(F), Y(F) \in$ $\mathcal{A}_{\sigma}(\Gamma)$.

Pour montrer qu'on a une action de $\mathcal{H}_{1}$ sur $\mathcal{A}_{\sigma}(\Gamma)$ il suffit de vérifier les relations (2.41) entre les opérateurs dans (2.44). Comme dans la démonstration de Proposition 2.5, on peut vérifier que $[Y, X](F)_{\alpha}=X(F)_{\alpha}$. De plus, puisque $X$ est une dérivation sur $\mathcal{M}$ et $\delta_{n}(F)_{\alpha}=X^{n-1}\left(\mu_{\alpha \sigma^{-1}}\right) \cdot F_{\alpha}$, on a

$$
\begin{aligned}
{\left[X, \delta_{n}\right](F)_{\alpha} } & =X\left(X^{n-1}\left(\mu_{\alpha \sigma^{-1}}\right) \cdot F_{\alpha}\right)-X^{n-1}\left(\mu_{\alpha \sigma^{-1}}\right) \cdot X\left(F_{\alpha}\right) \\
& =X\left(X^{n-1}\left(\mu_{\alpha \sigma^{-1}}\right)\right) \cdot F_{\alpha}=X^{n}\left(\mu_{\alpha \sigma^{-1}}\right) \cdot F_{\alpha}=\delta_{n+1}(F)_{\alpha}
\end{aligned}
$$

De même, pusique $\mu_{\alpha \sigma^{-1}} \in \mathcal{M}_{2}$ (voir $[5, \S 3]$ ) et $Y$ est une dérivation sur $\mathcal{M}$, on a

$$
\begin{aligned}
{\left[Y, \delta_{n}\right](F)_{\alpha} } & =Y\left(X^{n-1}\left(\mu_{\alpha \sigma^{-1}}\right) \cdot F_{\alpha}\right)-X^{n-1}\left(\mu_{\alpha \sigma^{-1}}\right) \cdot Y\left(F_{\alpha}\right) \\
& =Y\left(X^{n-1}\left(\mu_{\alpha \sigma^{-1}}\right)\right) \cdot F_{\alpha}=n X^{n-1}\left(\mu_{\alpha \sigma^{-1}}\right) \cdot F_{\alpha}=n \delta_{n}(F)_{\alpha} .
\end{aligned}
$$

Enfin, on peut vérifier aisément que $\left[\delta_{k}, \delta_{l}\right](F)_{\alpha}=0, \forall, k, l \geq 1$.

(b) Il suffit de vérifier (2.45) pour les générateurs $X, Y$ et $\delta_{1}$. Pour $F \in \mathcal{A}_{\sigma}(\Gamma)$, $G \in \mathcal{A}(\Gamma)$ et $\alpha \in G L_{2}^{+}(\mathbb{Q})$, on a

$$
\begin{aligned}
(X(F * G))_{\alpha} & =X\left((F * G)_{\alpha}\right) \\
& =\sum_{\beta \in \Gamma \backslash G L_{2}^{+}(\mathbb{Q})} X\left(F_{\beta \sigma} \cdot\left(G_{\alpha \sigma^{-1} \beta^{-1}} \mid \beta\right)\right) \\
& =\sum_{\beta \in \Gamma \backslash G L_{2}^{+}(\mathbb{Q})} X\left(F_{\beta \sigma}\right) \cdot\left(G_{\left.\alpha \sigma^{-1} \beta^{-1} \mid \beta\right)}+\sum_{\beta \in \Gamma \backslash G L_{2}^{+}(\mathbb{Q})} F_{\beta \sigma} \cdot X\left(G_{\alpha \sigma^{-1} \beta^{-1}} \mid \beta\right)\right. \\
& =(X(F), G)_{\alpha}+\sum_{\beta \in \Gamma \backslash G L_{2}^{+}(\mathbb{Q})} F_{\beta \sigma} \cdot X\left(G_{\alpha \sigma^{-1} \beta^{-1}}\right) \mid \beta \\
& +\sum_{\beta \in \Gamma \backslash G L_{2}^{+}(\mathbb{Q})} F_{\beta \sigma} \cdot \mu_{\beta} \cdot Y\left(G_{\alpha \sigma^{-1} \beta^{-1}}\right) \mid \beta \\
& =(X(F), G)_{\alpha}+(F, X(G))_{\alpha}+\sum_{\beta \in \Gamma \backslash G L_{2}^{+}(\mathbb{Q})} \delta_{1}(F)_{\beta \sigma} \cdot Y(G)_{\alpha \sigma^{-1} \beta^{-1}} \mid \beta \\
= & (X(F), G)_{\alpha}+(F, X(G))_{\alpha}+\left(\delta_{1}(F), Y(G)\right)_{\alpha} .
\end{aligned}
$$

Pour $\alpha, \beta \in G L_{2}^{+}(\mathbb{Q})$, il résulte de (2.21) que

$$
\mu_{\alpha \sigma^{-1}}=\mu_{\alpha \sigma^{-1} \beta^{-1} \beta}=\mu_{\alpha \sigma^{-1} \beta^{-1}} \mid \beta+\mu_{\beta} .
$$


Puisque $G \in \mathcal{A}(\Gamma)$, notons que $\delta_{1}(G)_{\alpha \sigma^{-1} \beta^{-1}}=\mu_{\alpha \sigma^{-1} \beta^{-1}} \cdot G_{\alpha \sigma^{-1} \beta^{-1}}$. Appliquant (2.50), on a :

$$
\begin{aligned}
\delta_{1}((F * G))_{\alpha} & =\mu_{\alpha \sigma^{-1}} \cdot(F * G)_{\alpha} \\
& =\sum_{\beta \in \Gamma \backslash G L_{2}^{+}(\mathbb{Q})} \mu_{\alpha \sigma^{-1}} \cdot\left(F_{\beta \sigma} \cdot\left(G_{\alpha \sigma^{-1} \beta^{-1}} \mid \beta\right)\right) \\
& =\sum_{\beta \in \Gamma \backslash G L_{2}^{+}(\mathbb{Q})}\left(\mu_{\beta} \cdot F_{\beta \sigma}\right) \cdot\left(G_{\alpha \sigma^{-1} \beta^{-1}} \mid \beta\right) \\
& +\sum_{\beta \in \Gamma \backslash G L_{2}^{+}(\mathbb{Q})} F_{\beta \sigma} \cdot\left(\mu_{\alpha \sigma^{-1} \beta^{-1}} \cdot G_{\alpha \sigma^{-1} \beta^{-1}}\right) \mid \beta \\
= & \left(\delta_{1}(F) * G\right)_{\alpha}+\left(F * \delta_{1}(G)\right)_{\alpha}
\end{aligned}
$$

Enfin, on peut vérifier aisément que $(Y(F * G))_{\alpha}=(Y(F) * G)_{\alpha}+(F * Y(G))_{\alpha}$.

Soit $\Gamma$ un sous-groupe principal de congruence de $S L_{2}(\mathbb{Z})$. Choisissons $f(z) \in \mathcal{M}_{k}(\Gamma)$ et $g(z) \in \mathcal{M}_{l}(\Gamma)$. D'après Zagier [11], on peut exprimer les crochets de Rankin-Cohen sur les formes modulaires comme $(\forall n \geq 0)$ :

$$
R C_{n}(f, g):=\sum_{r+s=n}(-1)^{r}\left(\begin{array}{c}
n+k-1 \\
s
\end{array}\right)\left(\begin{array}{c}
n+l-1 \\
r
\end{array}\right) D^{r} f(z) D^{s} g(z)
$$

où $D$ est l'opérateur différentiel défini comme $D:=\frac{1}{2 \pi i} \frac{d}{d z}$. Dans [6], les crochets de Rankin-Cohen sur l'algèbre $\mathcal{A}(\Gamma)$ ont été exprimés en termes de l'action de $\mathcal{H}_{1}$ sur $\mathcal{A}(\Gamma)$. De plus, les crochets de Rankin-Cohen réduits sur $\mathcal{A}(\Gamma)$ ont été définis par Yao [10, (II.6)] en utilisant l'action de $\mathfrak{h}_{0}$ sur $\mathcal{A}(\Gamma)$. Donc, on va utiliser l'action de $\mathfrak{h}_{0}$ sur $\mathcal{A}_{\sigma}(\Gamma)$ pour définir les crochets de Rankin-Cohen réduits sur les opérateurs tordus. Pour en savoir plus sur les liens entre les déformations de Rankin-Cohen et les algèbres de Hopf, voir [2], [8], [10].

Pour $k, l \in \mathbb{Z}$, posons $(2 Y+k)_{l}:=(2 Y+k)(2 Y+k+1) \ldots .(2 Y+k+l-1)$. Pour $F, G \in \mathcal{A}_{\sigma}(\Gamma)$ on définit les crochets de Rankin-Cohen réduits d'ordre $n \geq 0$ comme :

$$
\mathcal{R} \mathcal{C}_{n}(F, G):=\sum_{k=0}^{n}\left((-1)^{k} \frac{X^{k}}{k !}(2 Y+k)_{n-k}(F), \frac{X^{n-k}}{(n-k) !}(2 Y+n-k)_{k}(G)\right) .
$$

Proposition 2.8. Soient $F, G \in \mathcal{A}_{\sigma}(\Gamma)$. Alors, pour chaque $\alpha \in G L_{2}^{+}(\mathbb{Q})$ on a :

$$
\mathcal{R C}_{n}(F, G)_{\alpha}=\sum_{\beta \in \Gamma \backslash S L_{2}(\mathbb{Z})} R C_{n}\left(F_{\beta \sigma}, G_{\alpha \sigma^{-1} \beta^{-1}} \mid \sigma \beta\right) \quad \forall n \geq 0 .
$$


Démonstration. Par définition, on sait que $F, G$ sont des fonctions à supports finis. Alors, on a :

$$
\begin{aligned}
& \mathcal{R} \mathcal{C}_{n}(F, G)_{\alpha}= \sum_{k=0}^{n}\left((-1)^{k} \frac{X^{k}}{k !}(2 Y+k)_{n-k}(F), \frac{X^{n-k}}{(n-k) !}(2 Y+n-k)_{k}(G)\right)_{\alpha} \\
&=\sum_{k=0}^{n} \sum_{\beta \in \Gamma \backslash S L_{2}(\mathbb{Z})}(\left((-1)^{k} \frac{X^{k}}{k !}(2 Y+k)_{n-k}(F)_{\beta \sigma}\right) \\
&\left.\cdot\left(\frac{X^{n-k}}{(n-k) !}(2 Y+n-k)_{k}(G)_{\alpha \sigma^{-1} \beta^{-1}} \mid \sigma \beta\right)\right) \\
&=\sum_{\beta \in \Gamma \backslash S L_{2}(\mathbb{Z})} \sum_{k=0}^{n}\left(\left((-1)^{k} \frac{X^{k}}{k !}(2 Y+k)_{n-k}\left(F_{\beta \sigma}\right)\right)\right. \\
&\left.\cdot\left(\frac{X^{n-k}}{(n-k) !}(2 Y+n-k)_{k}\left(G_{\alpha \sigma^{-1} \beta^{-1}} \mid \sigma \beta\right)\right)\right)
\end{aligned}
$$

D'après Connes et Moscovici [6], la formule (2.52) de Zagier [11, § 1] pour les crochets de Rankin-Cohen sur les formes modulaires peut être écrite comme $(\forall n \geq 0)$ :

$$
\begin{array}{r}
R C_{n}(f, g)=\sum_{k=0}^{n}\left(\left((-1)^{k} \frac{X^{k}}{k !}(2 Y+k)_{n-k}(f)\right)\right. \\
\left.\cdot\left(\frac{X^{n-k}}{(n-k) !}(2 Y+n-k)_{k}(g)\right)\right) \forall f, g \in \mathcal{M}
\end{array}
$$

Combinant (2.55) et (2.56), on a

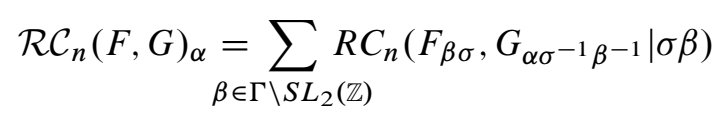

\section{Les opérateurs $X_{\tau}$}

Dans la section précédente, nous avons étudié les opérateurs de Hecke modulaires tordus par $\sigma$ pour chaque $\sigma \in S L_{2}(\mathbb{Z})$. Alors, à chaque niveau $\sigma \in S L_{2}(\mathbb{Z})$, on a une action de $\mathfrak{h}_{0}$ sur $\mathcal{A}_{\sigma}(\Gamma)$. Dans cette section, nous allons introduire des 
opérateurs entre les niveaux $\mathcal{A}_{\sigma}(\Gamma)$. Plus précisément, nous considérons la tour $\mathbb{A}_{\sigma}(\Gamma)=\bigoplus_{n \in \mathbb{Z}} \mathcal{A}_{\sigma(n)}(\Gamma)$, où $\sigma(n):=\left(\begin{array}{ll}1 & n \\ 0 & 1\end{array}\right) \cdot \sigma$. Nous montrons que l'action de ces opérateurs entre les niveaux de la tour $\mathbb{A}_{\sigma}(\Gamma)$ peut être exprimée en termes d'une algèbre de Hopf $\mathfrak{h}_{\mathbb{Z}} \supseteq \mathfrak{h}_{0}$ (voir (3.7)). Enfin, nous généralisons l'appariement sur $\mathcal{A}_{\sigma}(\Gamma)$ à un appariement sur $\mathbb{A}_{\sigma}(\Gamma)$ qui se comporte bien vis-à-vis de l'action de $\mathfrak{h}_{\mathbb{Z}}$.

Soit $\Gamma \subseteq S L_{2}(\mathbb{Z})$ un sous-groupe principal de congruence et soit $\tau \in S L_{2}(\mathbb{Z})$. Pour chaque $\sigma \in S L_{2}(\mathbb{Z})$ et $F \in \mathcal{A}_{\sigma}(\Gamma)$, nous définissons :

$$
\begin{gathered}
X_{\tau}(F): \Gamma \backslash G L_{2}^{+}(\mathbb{Q}) \longrightarrow \mathcal{M} \\
X_{\tau}(F)_{\alpha}:=X\left(F_{\alpha}\right) \mid \tau^{-1} \quad \forall \alpha \in G L_{2}^{+}(\mathbb{Q})
\end{gathered}
$$

Proposition 3.1. Soit $\Gamma \subseteq S L_{2}(\mathbb{Z})$ un sous-groupe principal de congruence.

(a) Fixons $\tau \in S L_{2}(\mathbb{Z})$. Alors, pour chaque $\sigma \in S L_{2}(\mathbb{Z}), X_{\tau}$ induit un morphisme

$$
X_{\tau}: \mathcal{A}_{\sigma}(\Gamma) \longrightarrow \mathcal{A}_{\tau \sigma}(\Gamma) .
$$

(b) Soient $\tau_{1}, \tau_{2} \in S L_{2}(\mathbb{Z})$ deux matrices telles que $\tau_{1} \tau_{2}=\tau_{2} \tau_{1}$. Alors, on a $\left[X_{\tau_{1}}, X_{\tau_{2}}\right]=0$.

Démonstration. (a) Pour chaque $\gamma \in \Gamma$, on a

$$
\begin{aligned}
X_{\tau}(F)_{\alpha \gamma} & =X\left(F_{\alpha \gamma}\right) \mid \tau^{-1} \\
& =X\left(F_{\alpha} \mid \sigma \gamma \sigma^{-1}\right) \mid \tau^{-1} \\
& =X\left(F_{\alpha} \mid \tau^{-1} \tau \sigma \gamma \sigma^{-1}\right) \mid \tau^{-1} \\
& \left.=\left(X\left(F_{\alpha}\right) \mid \tau^{-1}\right)\right) \mid \tau \sigma \gamma \sigma^{-1} \tau^{-1} \\
& =X_{\tau}(F)_{\alpha} \mid \tau \sigma \gamma \sigma^{-1} \tau^{-1}
\end{aligned}
$$

Donc, on voit que $X_{\tau}(F) \in \mathcal{A}_{\tau \sigma}$.

(b) Soit $F \in \mathcal{A}_{\sigma}(\Gamma)$. Alors, pour chaque $\alpha \in G L_{2}^{+}(\mathbb{Q})$, on a

$$
\begin{aligned}
\left(X_{\tau_{1}} X_{\tau_{2}}(F)\right)_{\alpha} & =X\left(X_{\tau_{2}}(F)_{\alpha}\right) \mid \tau_{1}^{-1} \\
& =X^{2}\left(F_{\alpha}\right) \mid \tau_{2}^{-1} \tau_{1}^{-1} \\
& =X^{2}\left(F_{\alpha}\right) \mid \tau_{1}^{-1} \tau_{2}^{-1} \\
& =\left(X_{\tau_{2}} X_{\tau_{1}}(F)\right)_{\alpha}
\end{aligned}
$$

Donc, $\left[X_{\tau_{1}}, X_{\tau_{2}}\right]=0$.

Pour chaque $m \in \mathbb{Z}$, posons $\rho_{m}:=\left(\begin{array}{cc}1 & m \\ 0 & 1\end{array}\right)$ et $X_{m}:=X_{\rho_{m}}$. De plus, pour chaque $\sigma \in S L_{2}(\mathbb{Z})$ et $n \in \mathbb{Z}$, posons $\sigma(n):=\rho_{n} \cdot \sigma$. Nous considérons la tour suivante :

$$
\mathbb{A}_{\sigma}(\Gamma):=\bigoplus_{n \in \mathbb{Z}} \mathcal{A}_{\sigma(n)}(\Gamma)
$$


Alors, $\mathbb{A}_{\sigma}(\Gamma)$ est un espace vectoriel gradué. Rappelons qu'un morphisme

$$
f: E=\bigoplus_{n \in \mathbb{Z}} E_{n} \longrightarrow E^{\prime}=\bigoplus_{n \in \mathbb{Z}} E_{n}^{\prime}
$$

des espaces gradués est dit homogène de degré $m$ si $f\left(E_{n}\right) \subseteq E_{n+m}^{\prime}$ pour tout $n \in \mathbb{Z}$ (voir par exemple [3, Chapitre 2]). Pour $m, n \in \mathbb{Z}$, notons que $\rho_{m} \cdot \rho_{n}=\rho_{n+m}$ et donc on a un morphisme $X_{m}=X_{\rho_{m}}: \mathcal{A}_{\sigma(n)}(\Gamma) \longrightarrow \mathcal{A}_{\sigma(n+m)}(\Gamma)$. Alors, pour chaque $m$, les morphismes $X_{m}: \mathcal{A}_{\sigma(n)}(\Gamma) \longrightarrow \mathcal{A}_{\sigma(n+m)}(\Gamma), \forall n \in \mathbb{Z}$ induisent un opérateur $X_{m}: \mathbb{A}_{\sigma}(\Gamma)=\bigoplus_{n \in \mathbb{Z}} \mathcal{A}_{\sigma(n)}(\Gamma) \longrightarrow \mathbb{A}_{\sigma}(\Gamma)=\bigoplus_{n \in \mathbb{Z}} \mathcal{A}_{\sigma(n)}(\Gamma)$ de degré $m$. De plus, soit $Z: \mathbb{A}_{\sigma}(\Gamma) \longrightarrow \mathbb{A}_{\sigma}(\Gamma)$ l'opérateur de degré 0 défini comme suit : pour $F \in \mathcal{A}_{\sigma(n)}(\Gamma)$, on pose :

$$
Z(F)_{\alpha}:=n F_{\alpha}+Y\left(F_{\alpha}\right) \quad \forall \alpha \in G L_{2}^{+}(\mathbb{Q})
$$

Nous considérons maintenant l'algèbre de Lie $\mathfrak{l}_{\mathbb{Z}}$ engendrée par les générateurs $\left\{Z, X_{m} \mid m \in \mathbb{Z}\right\}$ modulo les relations suivantes :

$$
\left[Z, X_{m}\right]=(m+1) X_{m} \quad\left[X_{m}, X_{m^{\prime}}\right]=0 \quad \forall m, m^{\prime} \in \mathbb{Z}
$$

En particulier, $\left[Z, X_{0}\right]=X_{0}$. Donc, l'algèbre de Lie $\mathfrak{l}_{0}$, qui agit sur $\mathcal{A}_{\sigma}(\Gamma)$, est contenue dans $\mathfrak{l}_{\mathbb{Z}}$. Nous allons montrer que $\mathbb{A}_{\sigma}(\Gamma)$ est munie d'une action de l'algèbre de Lie $\mathfrak{l}_{\mathbb{Z}}$.

Proposition 3.2. Soit $\Gamma$ un sous-groupe principal de congruence de $S L_{2}(\mathbb{Z})$. Fixons $\sigma \in S L_{2}(\mathbb{Z})$. Alors, $\mathbb{A}_{\sigma}(\Gamma)$ est munie d'une action de l'algèbre de Lie $\mathfrak{l}_{\mathbb{Z}}$ comme suit :

$$
\begin{aligned}
\left(X_{m}(F)\right)_{\alpha}=X\left(F_{\alpha}\right) \mid \rho_{m}^{-1} \quad Z(F)_{\alpha} & :=n F_{\alpha}+Y\left(F_{\alpha}\right) \\
\forall F & \in \mathcal{A}_{\sigma(n)}(\Gamma), \alpha \in G L_{2}^{+}(\mathbb{Q}), m, n \in \mathbb{Z}
\end{aligned}
$$

Démonstration. Il suffit de vérifier les relations (3.7) pour les opérateurs $Z, X_{m}$, $m \in \mathbb{Z}$. Comme une conséquence de Proposition 3.1(b), on sait que les opérateurs $X_{m}:=X_{\rho_{m}}, m \in \mathbb{Z}$ se commutent entre eux. De plus, pour $F \in \mathcal{A}_{\sigma(n)}(\Gamma)$ et $\alpha \in G L_{2}^{+}(\mathbb{Q})$, on a

$$
\begin{aligned}
\left(Z X_{m}(F)\right)_{\alpha} & =(n+m) X_{m}(F)_{\alpha}+Y X_{m}(F)_{\alpha} \\
& =(n+m) X\left(F_{\alpha}\right)\left|\rho_{m}^{-1}+Y X\left(F_{\alpha}\right)\right| \rho_{m}^{-1} \\
\left(X_{m} Z(F)\right)_{\alpha} & =X\left(Z(F)_{\alpha}\right) \mid \rho_{m}^{-1} \\
& =n X\left(F_{\alpha}\right)\left|\rho_{m}^{-1}+X Y\left(F_{\alpha}\right)\right| \rho_{m}^{-1}
\end{aligned}
$$

Puisque $[Y, X]=X$, il résulte de (3.9) que $\left[Z, X_{m}\right](F)_{\alpha}=m X_{m}(F)_{\alpha}+X_{m}(F)_{\alpha}=$ $(m+1) X_{m}(F)_{\alpha}$. 
Soit $\mathfrak{h}_{\mathbb{Z}}$ l'algèbre enveloppante de $\mathfrak{l}_{\mathbb{Z}}$. Donc, le coproduit $\Delta$ sur $\mathfrak{h}_{\mathbb{Z}}$ est défini comme suit :

$$
\Delta(Z)=Z \otimes 1+1 \otimes Z \quad \Delta\left(X_{m}\right)=X_{m} \otimes 1+1 \otimes X_{m} \quad \forall m \in \mathbb{Z}
$$

Alors, on a une action induite de $\mathfrak{h}_{\mathbb{Z}}$ sur $\mathbb{A}_{\sigma}(\Gamma)=\bigoplus_{n \in \mathbb{Z}} \mathcal{A}_{\sigma(n)}(\Gamma)$. On veut construire un appariement sur $\mathbb{A}_{\sigma}(\Gamma)$ qui se comporte bien vis-à-vis de l'action de $\mathfrak{h}_{\mathbb{Z}}$. Nous généralisons maintenant l'appariement défini dans (2.11).

Proposition 3.3. Soit $\Gamma$ un sous-groupe principal de congruence de $S L_{2}(\mathbb{Z})$. Fixons $\sigma \in S L_{2}(\mathbb{Z})$. Soient $\tau_{1}, \tau_{2} \in S L_{2}(\mathbb{Z})$ deux matrices telles que $\tau_{1} \tau_{2}=\tau_{2} \tau_{1}$. Alors, on a un appariement $(\ldots,-):, \mathcal{A}_{\tau_{1} \sigma}(\Gamma) \otimes \mathcal{A}_{\tau_{2} \sigma}(\Gamma) \longrightarrow \mathcal{A}_{\tau_{1} \tau_{2} \sigma}(\Gamma)$ défini comme suit $\left(\forall F \in \mathcal{A}_{\tau_{1} \sigma}(\Gamma), G \in \mathcal{A}_{\tau_{2} \sigma}(\Gamma), \alpha \in G L_{2}^{+}(\mathbb{Q})\right)$ :

$$
(F, G)_{\alpha}:=\sum_{\beta \in \Gamma \backslash S L_{2}(\mathbb{Z})}\left(F_{\beta \sigma} \mid \tau_{2}^{-1}\right) \cdot\left(G_{\alpha \sigma^{-1} \beta^{-1}} \mid \tau_{2} \sigma \beta \tau_{1}^{-1} \tau_{2}^{-1}\right)
$$

Démonstration. Prenons $\gamma \in \Gamma$. Alors, pour chaque $\beta \in S L_{2}(\mathbb{Z})$, on a

$$
\begin{aligned}
\left(G_{\alpha \sigma^{-1} \beta^{-1} \gamma^{-1}} \mid \tau_{2} \sigma \gamma \beta \tau_{1}^{-1} \tau_{2}^{-1}\right) & =\left(G_{\alpha \sigma^{-1} \beta^{-1}} \mid \tau_{2} \sigma \gamma^{-1} \sigma^{-1} \tau_{2}^{-1} \tau_{2} \sigma \gamma \beta \tau_{1}^{-1} \tau_{2}^{-1}\right) \\
& =\left(G_{\alpha \sigma^{-1} \beta^{-1}} \mid \tau_{2} \sigma \beta \tau_{1}^{-1} \tau_{2}^{-1}\right)
\end{aligned}
$$

et donc la somme dans (3.11) est bien définie. De plus, on a

$$
(F, G)_{\alpha \gamma}:=\sum_{\beta \in \Gamma \backslash S L_{2}(\mathbb{Z})}\left(F_{\beta \sigma} \mid \tau_{2}^{-1}\right) \cdot\left(G_{\alpha \gamma \sigma^{-1} \beta^{-1}} \mid \tau_{2} \sigma \beta \tau_{1}^{-1} \tau_{2}^{-1}\right)
$$

Dans (3.12), nous posons $\delta=\beta \sigma \gamma^{-1} \sigma^{-1}$. Puisque $F \in \mathcal{A}_{\tau_{1} \sigma}(\Gamma)$, on a $F_{\delta \sigma \gamma}=$ $F_{\delta \sigma} \mid \tau_{1} \sigma \gamma \sigma^{-1} \tau_{1}^{-1}$. Alors, on peut écrire (3.12) comme

$$
\begin{aligned}
(F, G)_{\alpha \gamma} & =\sum_{\beta \in \Gamma \backslash S L_{2}(\mathbb{Z})}\left(F_{\delta \sigma \gamma} \mid \tau_{2}^{-1}\right) \cdot\left(G_{\alpha \sigma^{-1} \delta^{-1}} \mid \tau_{2} \sigma \delta \sigma \gamma \sigma^{-1} \tau_{1}^{-1} \tau_{2}^{-1}\right) \\
& =\sum_{\beta \in \Gamma \backslash S L_{2}(\mathbb{Z})}\left(F_{\delta \sigma} \mid \tau_{1} \sigma \gamma \sigma^{-1} \tau_{1}^{-1} \tau_{2}^{-1}\right) \cdot\left(G_{\left.\alpha \sigma^{-1} \delta^{-1} \mid \tau_{2} \sigma \delta \sigma \gamma \sigma^{-1} \tau_{1}^{-1} \tau_{2}^{-1}\right)}\right. \\
& =\left(\sum_{\beta \in \Gamma \backslash S L_{2}(\mathbb{Z})}\left(F_{\delta \sigma} \mid \tau_{2}^{-1}\right) \cdot\left(G_{\alpha \sigma^{-1} \delta^{-1}} \mid \tau_{2} \sigma \delta \tau_{1}^{-1} \tau_{2}^{-1}\right)\right) \mid \tau_{1} \tau_{2} \sigma \gamma \sigma^{-1} \tau_{1}^{-1} \tau_{2}^{-1} \\
& =(F, G)_{\alpha} \mid \tau_{1} \tau_{2} \sigma \gamma \sigma^{-1} \tau_{1}^{-1} \tau_{2}^{-1}
\end{aligned}
$$

Donc, $(F, G) \in \mathcal{A}_{\tau_{1} \tau_{2} \sigma}(\Gamma)$.

En particulier, pour $n, n^{\prime} \in \mathbb{Z}$, on a un appariement $(\ldots, \ldots): \mathcal{A}_{\sigma(n)}(\Gamma) \otimes$ $\mathcal{A}_{\sigma\left(n^{\prime}\right)}(\Gamma) \longrightarrow \mathcal{A}_{\sigma\left(n+n^{\prime}\right)}(\Gamma)$. Puisque $\mathbb{A}_{\sigma}(\Gamma)=\bigoplus_{n \in \mathbb{Z}} \mathcal{A}_{\sigma(n)}(\Gamma)$, il est clair qu'on peut étendre l'appariement défini dans Proposition 3.3 à un appariement (__,_- ) : $\mathbb{A}_{\sigma}(\Gamma) \otimes \mathbb{A}_{\sigma}(\Gamma) \longrightarrow \mathbb{A}_{\sigma}(\Gamma)$. 
Lemme 3.4. Soit $\Gamma$ un sous-groupe principal de congruence de $S L_{2}(\mathbb{Z})$. Fixons $\sigma \in S L_{2}(\mathbb{Z})$. Soient $\tau_{1}, \tau_{2}, \tau_{3} \in S L_{2}(\mathbb{Z})$ trois matrices telles que $\tau_{i} \tau_{j}=\tau_{j} \tau_{i}, \forall i$, $j \in\{1,2,3\}$. Alors, pour chaque $F \in \mathcal{A}_{\tau_{1} \sigma}(\Gamma), G \in \mathcal{A}_{\tau_{2} \sigma}(\Gamma)$, on $a$ :

$$
X_{\tau_{3}}(F, G)=\left(X_{\tau_{3}}(F), G\right)+\left(F, X_{\tau_{3}}(G)\right)
$$

Démonstration. Par définition, pour chaque $\alpha \in G L_{2}^{+}(\mathbb{Q})$, on a :

$$
\begin{aligned}
& X_{\tau_{3}}(F, G)_{\alpha}=\sum_{\beta \in \Gamma \backslash S L_{2}(\mathbb{Z})} X\left(\left(F_{\beta \sigma} \mid \tau_{2}^{-1}\right) \cdot\left(G_{\alpha \sigma^{-1} \beta^{-1}} \mid \tau_{2} \sigma \beta \tau_{1}^{-1} \tau_{2}^{-1}\right)\right) \mid \tau_{3}^{-1} \\
& =\sum_{\beta \in \Gamma \backslash S L_{2}(\mathbb{Z})}\left(X\left(F_{\beta \sigma}\right) \mid \tau_{2}^{-1} \tau_{3}^{-1}\right) \cdot\left(G_{\alpha \sigma^{-1} \beta^{-1}} \mid \tau_{2} \sigma \beta \tau_{1}^{-1} \tau_{2}^{-1} \tau_{3}^{-1}\right) \\
& +\sum_{\beta \in \Gamma \backslash S L_{2}(\mathbb{Z})}\left(F_{\beta \sigma} \mid \tau_{2}^{-1} \tau_{3}^{-1}\right) \cdot\left(X\left(G_{\alpha \sigma^{-1} \beta^{-1}}\right) \mid \tau_{2} \sigma \beta \tau_{1}^{-1} \tau_{2}^{-1} \tau_{3}^{-1}\right)
\end{aligned}
$$

Notons que $X_{\tau_{3}}(F) \in \mathcal{A}_{\tau_{1} \tau_{3} \sigma}(\Gamma)$ et $X_{\tau_{3}}(G) \in \mathcal{A}_{\tau_{2} \tau_{3} \sigma}(\Gamma)$. Appliquant l'appariement défini dans Proposition 3.3, on a

$$
\begin{aligned}
\left(X_{\tau_{3}}(F), G\right)_{\alpha} & =\sum_{\beta \in \Gamma \backslash S L_{2}(\mathbb{Z})}\left(X_{\tau_{3}}(F)_{\beta \sigma} \mid \tau_{2}^{-1}\right) \cdot\left(G_{\alpha \sigma^{-1} \beta^{-1}} \mid \tau_{2} \sigma \beta \tau_{1}^{-1} \tau_{2}^{-1} \tau_{3}^{-1}\right) \\
& =\sum_{\beta \in \Gamma \backslash S L_{2}(\mathbb{Z})}\left(X\left(F_{\beta \sigma}\right) \mid \tau_{2}^{-1} \tau_{3}^{-1}\right) \cdot\left(G_{\alpha \sigma^{-1} \beta^{-1}} \mid \tau_{2} \sigma \beta \tau_{1}^{-1} \tau_{2}^{-1} \tau_{3}^{-1}\right) \\
\left(F, X_{\tau_{3}}(G)\right)_{\alpha} & =\sum_{\beta \in \Gamma \backslash S L_{2}(\mathbb{Z})}\left(F_{\beta \sigma} \mid \tau_{2}^{-1} \tau_{3}^{-1}\right) \cdot\left(X_{\tau_{3}}(G)_{\alpha \sigma^{-1} \beta^{-1}} \mid \tau_{2} \tau_{3} \sigma \beta \tau_{1}^{-1} \tau_{2}^{-1} \tau_{3}^{-1}\right) \\
& =\sum_{\beta \in \Gamma \backslash S L_{2}(\mathbb{Z})}\left(F_{\beta \sigma} \mid \tau_{2}^{-1} \tau_{3}^{-1}\right) \cdot\left(X\left(G_{\alpha \sigma^{-1} \beta^{-1}}\right) \mid \tau_{3}^{-1} \tau_{2} \tau_{3} \sigma \beta \tau_{1}^{-1} \tau_{2}^{-1} \tau_{3}^{-1}\right) \\
& =\sum_{\beta \in \Gamma \backslash S L_{2}(\mathbb{Z})}\left(F_{\beta \sigma} \mid \tau_{2}^{-1} \tau_{3}^{-1}\right) \cdot\left(X\left(G_{\alpha \sigma^{-1} \beta^{-1}}\right) \mid \tau_{2} \sigma \beta \tau_{1}^{-1} \tau_{2}^{-1} \tau_{3}^{-1}\right)
\end{aligned}
$$

Ceci montre le résultat.

Enfin, nous montrons que l'appariement $(\ldots, \ldots): \mathbb{A}_{\sigma}(\Gamma) \otimes \mathbb{A}_{\sigma}(\Gamma) \longrightarrow \mathbb{A}_{\sigma}(\Gamma)$ se comporte bien vis-à-vis de l'action de $\mathfrak{h}_{\mathbb{Z}} \operatorname{sur} \mathbb{A}_{\sigma}(\Gamma)$.

Proposition 3.5. Soit $\Gamma$ un sous-groupe principal de congruence de $S L_{2}(\mathbb{Z})$ et soit $\sigma \in S L_{2}(\mathbb{Z})$. Pour chaque $h \in \mathfrak{h}_{\mathbb{Z}}$, posons $\Delta(h)=\sum h_{(1)} \otimes h_{(2)}$. Alors, pour chaque $F, G \in \mathbb{A}_{\sigma}(\Gamma)$, on a

$$
h(F, G)=\sum\left(h_{(1)}(F), h_{(2)}(G)\right)
$$


Démonstration. Choisissons $n, n^{\prime} \in \mathbb{Z}$. On peut supposer que $F \in \mathcal{A}_{\sigma(n)}(\Gamma)$ et $G \in \mathcal{A}_{\sigma\left(n^{\prime}\right)}(\Gamma)$. Il suffit de vérifier (3.17) pour les générateurs $\left\{Z, X_{m} \mid m \in \mathbb{Z}\right\}$ de l'algèbre de Hopf $\mathfrak{h}_{\mathbb{Z}}$. Pour les générateurs $X_{m}, m \in \mathbb{Z}$, ceci est une conséquence du Lemme 3.4. Puisque $\Delta(Z)=Z \otimes 1+1 \otimes Z$, il reste à montrer que

$$
Z(F, G)=(Z(F), G)+(F, Z(G)) \quad \forall F \in \mathcal{A}_{\sigma(n)}(\Gamma), G \in \mathcal{A}_{\sigma\left(n^{\prime}\right)}(\Gamma)
$$

Appliquant Proposition 3.3, il est clair que $(F, G) \in \mathcal{A}_{\sigma\left(n+n^{\prime}\right)}(\Gamma)$. Alors, pour chaque $\alpha \in G L_{2}^{+}(\mathbb{Q})$, on a

$$
\begin{aligned}
& Z(F, G)_{\alpha}=\left(n+n^{\prime}\right)(F, G)_{\alpha}+Y(F, G)_{\alpha} \\
&=\left(n+n^{\prime}\right) \sum_{\beta \in \Gamma \backslash S L_{2}(\mathbb{Z})}\left(( F _ { \beta \sigma } | \rho _ { n ^ { \prime } } ^ { - 1 } ) \cdot \left(G_{\left.\left.\alpha \sigma^{-1} \beta^{-1} \mid \rho_{n^{\prime}} \sigma \beta \rho_{n}^{-1} \rho_{n^{\prime}}^{-1}\right)\right)}\right.\right. \\
& \quad+\sum_{\beta \in \Gamma \backslash S L_{2}(\mathbb{Z})} Y\left(( F _ { \beta \sigma } | \rho _ { n ^ { \prime } } ^ { - 1 } ) \cdot \left(G_{\left.\left.\alpha \sigma^{-1} \beta^{-1} \mid \rho_{n^{\prime}} \sigma \beta \rho_{n}^{-1} \rho_{n^{\prime}}^{-1}\right)\right)}\right.\right. \\
&=\sum_{\beta \in \Gamma \backslash S L_{2}(\mathbb{Z})}\left(\left(n F_{\beta \sigma}+Y\left(F_{\beta \sigma}\right)\right) \mid \rho_{n^{\prime}}^{-1}\right) \cdot\left(G_{\alpha \sigma^{-1} \beta^{-1}} \mid \rho_{n^{\prime}} \sigma \beta \rho_{n}^{-1} \rho_{n^{\prime}}^{-1}\right) \\
& \quad+\sum_{\beta \in \Gamma \backslash S L_{2}(\mathbb{Z})}\left(F_{\beta \sigma} \mid \rho_{n^{\prime}}^{-1}\right) \cdot\left(\left(n^{\prime} G_{\alpha \sigma^{-1} \beta^{-1}}+Y\left(G_{\left.\left.\left.\alpha \sigma^{-1} \beta^{-1}\right)\right) \mid \rho_{n^{\prime}} \sigma \beta \rho_{n}^{-1} \rho_{n^{\prime}}^{-1}\right)}\right.\right.\right. \\
&=\sum_{\beta \in \Gamma \backslash S L_{2}(\mathbb{Z})}\left(Z(F)_{\beta \sigma} \mid \rho_{n^{\prime}}^{-1}\right) \cdot\left(G_{\alpha \sigma^{-1} \beta^{-1}} \mid \rho_{n^{\prime}} \sigma \beta \rho_{n}^{-1} \rho_{n^{\prime}}^{-1}\right) \\
& \quad+\sum_{\beta \in \Gamma \backslash S L_{2}(\mathbb{Z})}\left(F_{\beta \sigma} \mid \rho_{n^{\prime}}^{-1}\right) \cdot\left(Z(G)_{\left.\alpha \sigma^{-1} \beta^{-1} \mid \rho_{n^{\prime}} \sigma \beta \rho_{n}^{-1} \rho_{n^{\prime}}^{-1}\right)}\right. \\
&=(Z(F), G)_{\alpha}+(F, Z(G))_{\alpha}
\end{aligned}
$$

\section{Références}

[1] A. Banerjee, Hopf Action and Rankin-Cohen Brackets on an Archimedean Complex, J. Noncommut. Geom. 5 (2011), no. 3, 401-421. Zbl 1263.11051 MR 2817645

[2] P. Bieliavsky, X. Tang and Y.-J. Yao, Rankin-Cohen brackets and formal quantization, Adv. Math. 212 (2007). no. 1, 293-314. Zbl 1123.53049 MR 2319770

[3] N. Bourbaki, Éléments de mathématique. Algèbre. Chapitres 1 à 3, Hermann, Paris, 1970. Zbl 0211.02401 MR 274237 
[4] A. Connes and H. Moscovici, Hopf algebras, cyclic cohomology and the transverse index theorem, Comm. Math. Phys., 198 (1998),199-246. Zbl 0940.58005 MR 1657389

[5] A. Connes and H. Moscovici, Modular Hecke algebras and their Hopf symmetry, Moscow Math Journal, 4 (2004), 67-109. Zbl 1122.11023 MR 2074984

[6] A. Connes and H. Moscovici, Rankin-Cohen brackets and the Hopf algebra of transverse geometry. Moscow Math. Journal. 4 (2004), 111-130. Zbl 1122.11024 MR 2074985

[7] M. Crainic, Cyclic cohomology of Hopf algebras, and a non-commutative Chern-Weil theory, Preprint, 1999. arXiv:math/9812113v3

[8] R. Rochberg, X. Tang abd Y.-J. Yao, A survey on Rankin-Cohen deformations in Perspectives on noncommutative geometry, 133-151, Fields Inst. Commun., 61, Amer. Math. Soc., Providence, RI, 2011. Zbl 1287.46054 MR 2838685

[9] G. Shimura, Introduction to the arithmetic theory of automorphic functions, Publ. Math. Soc. Japan, 11, Iwanami Shoten/Princeton Univ. Press, Tokyo/Princeton, NJ, 1971. Zbl 0221.10029 MR 314766

[10] Y.-J. Yao, Autour des Déformations de Rankin-Cohen, Thèse de Doctorat, École Polytechnique, 2007.

[11] D. Zagier, Modular forms and differential operators, K.G. Ramanathan memorial issue, Proc. Indian Acad. Sci. Math 104 (1994), no. 1, 57-75. Zbl 0806.11022 MR 1280058

Received 12 April, 2014

A. Banerjee, Department of Mathematics, Indian Institute of Science, Bangalore 560012 , India

E-mail: abhishekbanerjee1313@gmail.com 